\title{
Jordanian quantum spheres
}

\author{
R. Chakrabarti ${ }^{1}$ and J. Segar ${ }^{2}$ \\ ${ }^{1}$ Department of Theoretical Physics, University of Madras, \\ Guindy Campus, Madras-600 025, India. \\ ${ }^{2}$ Department of Physics, R. K. M. Vivekananda College, Madras-600 004, India.
}

\begin{abstract}
We introduce and investigate a one parameter family of quantum spaces invariant under the left (right) coactions of the group-like element $\mathcal{T}_{h}^{(j=1)}$ of the Jordanian function algebra $\operatorname{Fun}_{h}(S L(2))$. These spaces may be regarded as Jordanian quantization of the two-dimensional spheres.
\end{abstract}




\section{Introduction}

Noncommutative quantum spheres were first studied by Podles [1] by considering a oneparameter family of quantum homogeneous spaces for the standard $q$-deformed quantum group $S L_{q}(2)$. Exterior algebras of differential forms on these $q$-deformed quantum spaces were investigated [2], [3] and classification of covariant differential calculi on Podles quantum $q$-spheres was obtained [4]. Orthogonal bases for the algebra of functions on Podles quantum $q$-spheres were determined [5] in terms of the big $q$-Jacobi polynomials. An elegant description of the $q$-spherical functions was obtained [6] by considering left and right invariances in the infinitesimal sense with respect to twisted primitive elements of the $q$-deformed universal enveloping algebra $U_{q}(s l(2))$.

On the other hand it is well-known that the Lie group $S L(2)$ admits [7] two distinct quantum group deformations with central quantum determinant: the standard $q$-deformation $\operatorname{Fun}_{q}(S L(2))$ and the Jordanian deformation $\operatorname{Fun}_{h}(S L(2))$ [8]. On the quantum algebra level, the Jordanian deformation $U_{h}(\operatorname{sl}(2))$ of the corresponding classical enveloping algebra was constructed [9]. The differential calculas on the Jordanian $h$-deformed quantum plane was investigated [10], and the classification of the bicovariant diffential calculi on the Jordanian function algebra $\operatorname{Fun}_{h}(S L(2))$ was obtained [11]. Using a contraction technique developed earlier [12, 13, a general recipe for obtaining the finite dimensional group-like elements $\mathcal{T}_{h}^{(j)}$ of the function algebra $\operatorname{Fun}_{h}(S L(2))$ was described in [14.

In the present work our starting point is the explicit construction of the matrix $\mathcal{T}_{h}^{(j=1)}$ obtained in [14]. Investigating the left (right) coaction of $\mathcal{T}_{h}^{(j=1)}$ on quantum spaces with three noncommuting generators we obtain a one parameter family of algebras invariant under the coaction. These invariant quantum spaces may be regarded as Jordanian deformation of the two-dimensional spheres. We demonostrate that at the algebraic level there exist an invertible map between the quantum spaces invariant under the left and the right coactions of the function algebra $\operatorname{Fun}_{h}(S L(2))$ respectively. Moreover, the quantum spaces may be realized as the subalgebras of the function algebra $F u n_{h}(S L(2))$. Lastly, following [6], the above embedding may be obtained by using the infinitesimal invariance of the generating elements with respect to a corresponding twisted primitive element of the dual Jordanian enveloping algebra $U_{h}(s l(2))$.

A motivation for the present work is the recent interest in formulating field theory on noncommutative spaces, both from the point of view of finding new insights into the renormalization problem [15]-[19]; and the realization that such noncommutative spaces are induced by certain sectors of string theory, particularly open strings ending on $D$-branes with a background $B$ field [20]-22]. We, therefore, expect exploration of new noncommutative spaces to be fruitful. 


\section{The left and the right coactions of the $\mathcal{T}_{h}^{(j=1)}$ matrix}

The defining relations for the Jordanian deformed $U_{h}(s l(2))$ algebra read [9]

$$
\begin{aligned}
& {\left[H, T^{ \pm 1}\right]=T^{ \pm 2}-1,} \\
& {[H, Y]=-\frac{1}{2}\left(Y\left(T+T^{-1}\right)+\left(T+T^{-1}\right) Y\right),} \\
& {\left[T^{ \pm 1}, Y\right]= \pm \frac{h}{2}\left(H T^{ \pm 1}+T^{ \pm 1} H\right) .}
\end{aligned}
$$

The corresponding coproduct $(\triangle)$, counit $(\varepsilon)$ and the antipode $(S)$ maps are given by

$$
\begin{aligned}
& \triangle\left(T^{ \pm 1}\right)=T^{ \pm 1} \otimes T^{ \pm 1}, \triangle(Y)=Y \otimes T+T^{-1} \otimes Y, \triangle(H)=H \otimes T+T^{-1} \otimes H, \\
& \varepsilon\left(T^{ \pm 1}\right)=1, \quad \varepsilon(Y)=\varepsilon(H)=0, \\
& S\left(T^{ \pm 1}\right)=T^{\mp 1}, \quad S(Y)=-T Y T^{-1}, \quad S(H)=-T H T^{-1} .
\end{aligned}
$$

The dual Jordanian function algebra $\mathrm{Fun}_{h}(S L(2))$ is generated by the elements of the matrix $\mathcal{T}_{h}^{\left(j=\frac{1}{2}\right)}=\left(\begin{array}{ll}a & b \\ c & d\end{array}\right)$, obeying the relations

$$
\begin{aligned}
& {[a, b]=h\left(a^{2}-1\right), \quad[a, c]=-h c^{2},} \\
& {[b, d]=-h\left(d^{2}-1\right), \quad[c, d]=h c^{2},} \\
& {[a, d]=h a c-h d c, \quad[b, c]=-h a c-h c d,}
\end{aligned}
$$

where the central determinant $D=a d-b c-h a c$ satisfies the condition

$$
D=1 .
$$

The coalgebraic properties for the generating elements $(a, b, c, d)$ may be summarized as

$$
\begin{aligned}
& \triangle\left(\begin{array}{ll}
a & b \\
c & d
\end{array}\right)=\left(\begin{array}{ll}
a & b \\
c & d
\end{array}\right) \dot{\otimes}\left(\begin{array}{ll}
a & b \\
c & d
\end{array}\right), \\
& \varepsilon\left(\begin{array}{ll}
a & b \\
c & d
\end{array}\right)=\left(\begin{array}{ll}
1 & 0 \\
0 & 1
\end{array}\right), \\
& S\left(\begin{array}{ll}
a & b \\
c & d
\end{array}\right)=\left(\begin{array}{cc}
d-h c & -b+h(a-d)+h^{2} c \\
-c & a+h c
\end{array}\right) .
\end{aligned}
$$

A recipe based on a contraction procedure has been provided in [14 for obtaining arbitrary finite dimensional group-like elements $\mathcal{T}_{h}^{(j)}$ of the $\operatorname{Fun}_{h}(S L(2))$ algebra. These $\mathcal{T}_{h}^{(j)}$ matrices may be regarded as the finite dimensional representations of the dual form between the 
Jordanian Hopf algebras Fun $\operatorname{Fu}_{h}(S L(2))$ and $U_{h}(s l(2))$. Using the determinantal condition (2.4), here we reproduce the explicit determination of the $\mathcal{T}_{h}^{(j=1)}$ obtained in [14]:

$$
\mathcal{T}_{h}^{(j=1)}=\left(\begin{array}{ccc}
a^{2}+\frac{1}{4} h^{2} c^{2} & 2 a b-h a^{2}+h+\frac{1}{2} h^{2} c d & 2 b^{2}-\frac{3}{2} h^{2} a^{2}+\frac{1}{2} h^{2} d^{2} \\
& -\frac{1}{4} h^{3} c^{2} & +h^{2}-\frac{3}{8} h^{4} c^{2} \\
a c+\frac{1}{2} h c^{2} & 1+2 b c+h a c+h c d & 2 b d+h d^{2}-h-\frac{3}{2} h^{2} a c \\
& -\frac{1}{2} h^{2} c^{2} & -\frac{3}{4} h^{3} c^{2} \\
\frac{1}{2} c^{2} & c d-\frac{1}{2} h c^{2} & d^{2}-\frac{3}{4} h^{2} c^{2}
\end{array}\right) .
$$

The group-like property of the Jordanian monodromy matrix $\mathcal{T}_{h}^{(j=1)}$ is manifest in its coproduct relation

$$
\triangle\left(\mathcal{T}_{h}^{(j=1)}\right)=\mathcal{T}_{h}^{(j=1)} \dot{\otimes} \mathcal{T}_{h}^{(j=1)}
$$

We define a left coaction $\phi_{\mathrm{L}}$ acting on the generators of a quantum space $\left(\mathrm{x}_{\mathrm{i}} \mid \mathrm{i}=-1,0,1\right)$ by the relation

$$
\phi_{\mathrm{L}}\left(\mathrm{x}_{\mathrm{i}}\right)=\sum_{\mathrm{j}=-1}^{1}\left(\mathcal{T}_{h}^{(j=1)}\right)_{\mathrm{ij}} \otimes \mathrm{x}_{\mathrm{j}} \quad \text { for } \quad \mathrm{i}=(-1,0,1) .
$$

As a consequence of the coproduct property (2.7) the coaction $\phi_{\mathrm{L}}$ satisfies the identity

$$
\left(\mathrm{id} \otimes \phi_{\mathrm{L}}\right) \circ \phi_{\mathrm{L}}\left(\mathrm{x}_{\mathrm{i}}\right)=(\triangle \otimes \mathrm{id}) \circ \phi_{\mathrm{L}}\left(\mathrm{x}_{\mathrm{i}}\right) \quad \text { for } \quad \mathrm{i}=(-1,0,1)
$$

For two complex numbers $(k, \beta)$, where $\beta \neq 0$, we denote by $\mathcal{X}_{(h ; k, \beta)}$ the algebra with the noncommuting generators $\left(x_{i} \mid i=-1,0,1\right)$ obeying the defining relations:

$$
\begin{aligned}
\mathrm{x}_{0}{ }^{2}-\mathrm{x}_{-1} \mathrm{x}_{1}-\mathrm{x}_{1} \mathrm{x}_{-1}+8 h^{2} \mathrm{x}_{1}^{2}+8 k h^{2} \mathrm{x}_{1} & =\beta \\
\mathrm{x}_{-1} \mathrm{x}_{0}-\mathrm{x}_{0} \mathrm{x}_{-1}+h \mathrm{x}_{-1} \mathrm{x}_{1}+3 h \mathrm{x}_{1} \mathrm{x}_{-1}+\frac{1}{2} h^{2} \mathrm{x}_{0} \mathrm{x}_{1}-\frac{9}{2} h^{2} \mathrm{x}_{1} \mathrm{x}_{0} & \\
-6 h^{3} \mathrm{x}_{1}^{2}+4 k h \mathrm{x}_{-1}-4 k h^{2} \mathrm{x}_{0}-6 k h^{3} \mathrm{x}_{1} & =0 \\
\mathrm{x}_{-1} \mathrm{x}_{1}-\mathrm{x}_{1} \mathrm{x}_{-1}+2 h \mathrm{x}_{0} \mathrm{x}_{1}+2 h \mathrm{x}_{1} \mathrm{x}_{0}+4 k h \mathrm{x}_{0} & =0 \\
\mathrm{x}_{0} \mathrm{x}_{1}-\mathrm{x}_{1} \mathrm{x}_{0}+4 h \mathrm{x}_{1}{ }^{2}+4 k h \mathrm{x}_{1} & =0
\end{aligned}
$$

The coaction $\phi_{\mathrm{L}}$ defined by (2.8) preserves the relations (2.10) and is an algebra homomorphism. The homomorphism $\phi_{\mathbf{L}}: \mathcal{X}_{(h ; k, \beta)} \rightarrow \operatorname{Fun}_{h}(S L(2)) \otimes \mathcal{X}_{(h ; k, \beta)}$ endows the algebra $\mathcal{X}_{(h ; k, \beta)}$ with the structure of a left $\operatorname{Fun}_{h}(S L(2))$ comodule.

In a similar way a right coaction $\phi_{\mathrm{R}}$ acting on the generating elements $\left(\mathrm{y}_{\mathrm{i}} \mid \mathrm{i}=-1,0,1\right)$ of a right quantum space for the funtion algebra $F u n_{h}(S L(2))$ may be defined as

$$
\phi_{\mathrm{R}}\left(\mathrm{y}_{\mathrm{i}}\right)=\sum_{\mathrm{j}=-1}^{1} \mathrm{y}_{\mathrm{j}} \otimes\left(\mathcal{T}_{h}^{(j=1)}\right)_{\mathrm{ji}} \quad \text { for } \quad \mathrm{i}=(-1,0,1) .
$$


The group-like coproduct property (2.7) now requires the right coaction $\phi_{\mathrm{R}}$ to satisfy the identity:

$$
\left(\phi_{\mathrm{R}} \otimes \mathrm{id}\right) \circ \phi_{\mathrm{R}}\left(\mathrm{y}_{\mathrm{i}}\right)=(\mathrm{id} \otimes \triangle) \circ \phi_{\mathrm{R}}\left(\mathrm{y}_{\mathrm{i}}\right) \text { for } \mathrm{i}=(-1,0,1) .
$$

The noncommuting generating elements $\left(y_{i} \mid i=-1,0,1\right)$ of the right quantum space furnish the algebra $\mathcal{Y}_{\left(h ; k^{\prime}, \beta^{\prime}\right)}$ defined by the relations

$$
\begin{aligned}
\mathrm{y}_{0}^{2}-\mathrm{y}_{-1} \mathrm{y}_{1}-\mathrm{y}_{1} \mathrm{y}_{-1}+4 h^{2} \mathrm{y}_{-1}{ }^{2}-8 k^{\prime} h^{2} \mathrm{y}_{-1} & =\beta^{\prime} \\
\mathrm{y}_{-1} \mathrm{y}_{0}-\mathrm{y}_{0} \mathrm{y}_{-1}-4 h \mathrm{y}_{-1}^{2}+4 k^{\prime} h \mathrm{y}_{-1} & =0 \\
\mathrm{y}_{-1} \mathrm{y}_{1}-\mathrm{y}_{1} \mathrm{y}_{-1}-2 h \mathrm{y}_{-1} \mathrm{y}_{0}-2 h \mathrm{y}_{0} \mathrm{y}_{-1}+4 k^{\prime} h \mathrm{y}_{0} & =0 \\
\mathrm{y}_{0} \mathrm{y}_{1}-\mathrm{y}_{1} \mathrm{y}_{0}-h \mathrm{y}_{-1} \mathrm{y}_{1}-3 h \mathrm{y}_{1} \mathrm{y}_{-1}-\frac{3}{2} h^{2} \mathrm{y}_{-1} \mathrm{y}_{0}-\frac{5}{2} h^{2} \mathrm{y}_{0} \mathrm{y}_{-1} & \\
-2 h^{3} \mathrm{y}_{-1}{ }^{2}+4 k^{\prime} h \mathrm{y}_{1}+4 k^{\prime} h^{2} \mathrm{y}_{0}+2 k^{\prime} h^{3} \mathrm{y}_{-1} & =0
\end{aligned}
$$

The parameters $\left(k^{\prime}, \beta^{\prime}\right)$, where $\beta^{\prime} \neq 0$, may be in general distinct from the parameters $(k, \beta)$ introduced in (2.10). The defining relations (2.13) are preserved under the coaction (2.11), which acts as an algebra homomorphism mapping $\mathcal{Y}_{\left(h ; k^{\prime}, \beta^{\prime}\right)} \rightarrow \mathcal{Y}_{\left(h ; k^{\prime}, \beta^{\prime}\right)} \otimes F u n_{h}(S L(2))$. The left and the right quantum spaces $\mathcal{X}_{(h ; k, \beta)}$ and $\mathcal{Y}_{\left(h ; k^{\prime}, \beta^{\prime}\right)}$ defined by the relations (2.10) and (2.13) respectively, may be mapped to each other at the algebraic level. We will discuss these mappings at the end of the present section.

Under the scaling of the generators $x_{i} \rightarrow c^{-1} x_{i}$, it follows from (2.10) that the defining relations of $\mathcal{X}_{(h ; k, \beta)}$ go into that of $\mathcal{X}_{\left(h ; c k, c^{2} \beta\right)}$. Consequently these quantum spaces are isomorphic to each other for any complex number $c \neq 0$. All of these equivalent quantum spaces may be referred to as left spheres. Using this isomorphism we may remove one superfluous parameter of the quantum space $\mathcal{X}_{(h ; k, \beta)}$. Similar argument holds for the right quantum space $\mathcal{Y}_{\left(h ; k^{\prime}, \beta^{\prime}\right)}$ defined by the relations (2.13). The scaling freedom may again be utilized to eliminate one redundant parameter in $\mathcal{Y}_{\left(h ; k^{\prime}, \beta^{\prime}\right)}$.

The algebras $\mathcal{X}_{(h ; k, \beta)}$ and $\mathcal{Y}_{\left(h ; k^{\prime}, \beta^{\prime}\right)}$ may be realized by embedding them in the function algebra $\operatorname{Fun}_{h}(S L(2))$. The generating elements of the $\mathcal{X}_{(h ; k, \beta)}$ algebra may be represented using the linear combinations of the matrix elements of the monodromy matrix $\mathcal{T}_{h}^{(j=1)}$ given in (2.6):

$$
\begin{aligned}
\mathrm{x}_{-1}= & k\left(a^{2}+\frac{1}{4} h^{2} c^{2}\right)+\rho\left(2 a b-h a^{2}+h+\frac{1}{2} h^{2} c d-\frac{1}{4} h^{3} c^{2}\right) \\
& -k\left(2 b^{2}-\frac{3}{2} h^{2} a^{2}+\frac{1}{2} h^{2} d^{2}+h^{2}-\frac{3}{8} h^{4} c^{2}\right), \\
\mathrm{x}_{0}= & k\left(a c+\frac{1}{2} h c^{2}\right)+\rho\left(1+2 b c+h a c+h c d-\frac{1}{2} h^{2} c^{2}\right) \\
& -k\left(2 b d+h d^{2}-h-\frac{3}{2} h^{2} a c-\frac{3}{4} h^{3} c^{2}\right), \\
\mathrm{x}_{1}= & \frac{1}{2} k c^{2}+\rho\left(c d-\frac{1}{2} h c^{2}\right)-k\left(d^{2}-\frac{3}{4} h^{2} c^{2}\right) .
\end{aligned}
$$


The representation (2.14) satisfies the defining relations (2.10). The paramater $\beta$ is now given by

$$
\beta=\rho^{2}+2 k^{2} .
$$

The representation (2.14) is particularly simple if we select previously mentioned scaling parameter $\mathrm{c}=\rho^{-1}$ and consider the limiting value $\rho \rightarrow \infty$ for a finite value of $k$. The consequent algebra $\mathcal{X}_{(h ; 0,1)}$ has an embedding in the function algebra $F_{u n}(S L(2))$ à la (2.14) as follows:

$$
\begin{aligned}
\mathrm{x}_{-1} & =2 a b-h a^{2}+h+\frac{1}{2} h^{2} c d-\frac{1}{4} h^{3} c^{2} \\
\mathrm{x}_{0} & =1+2 b c+h a c+h c d-\frac{1}{2} h^{2} c^{2} \\
\mathrm{x}_{1} & =c d-\frac{1}{2} h c^{2} .
\end{aligned}
$$

The group-like coproduct property (2.7) may be used to prove the following coproduct structure for the embedding (2.14)

$$
\triangle\left(\mathcal{X}_{(h ; k, \beta)}\right) \subseteq \operatorname{Fun}_{h}(S L(2)) \otimes \mathcal{X}_{(h ; k, \beta)} .
$$

Similarly the generators $\left(y_{i} \mid i=-1,0,1\right)$ of the right quantum space $\mathcal{Y}_{\left(h ; k^{\prime}, \beta^{\prime}\right)}$ may also be represented as linear combinations of the matrix elements of the group-like construct $\mathcal{T}_{h}^{(j=1)}$ given in (2.6). The embedding is implemented as follows:

$$
\begin{aligned}
\mathrm{y}_{-1}= & k^{\prime}\left(a^{2}+\frac{1}{4} h^{2} c^{2}\right)+\rho^{\prime}\left(a c+\frac{1}{2} h c^{2}\right)-\frac{1}{2} k^{\prime} c^{2}, \\
\mathrm{y}_{0}= & k^{\prime}\left(2 a b-h a^{2}+h+\frac{1}{2} h^{2} c d-\frac{1}{4} h^{3} c^{2}\right) \\
& +\rho^{\prime}\left(1+2 b c+h a c+h c d-\frac{1}{2} h^{2} c^{2}\right)-k^{\prime}\left(c d-\frac{1}{2} h c^{2}\right), \\
\mathrm{y}_{1}= & k^{\prime}\left(2 b^{2}-\frac{3}{2} h^{2} a^{2}+\frac{1}{2} h^{2} d^{2}+h^{2}-\frac{3}{8} h^{4} c^{2}\right) \\
& +\rho^{\prime}\left(2 b d+h d^{2}-h-\frac{3}{2} h^{2} a c-\frac{3}{4} h^{3} c^{2}\right)-k^{\prime}\left(d^{2}-\frac{3}{4} h^{2} c^{2}\right) .
\end{aligned}
$$

The representation (2.18) obeys the defining algebraic properties (2.13), and yields the following value of the parameter $\beta^{\prime}$ :

$$
\beta^{\prime}=\rho^{\prime 2}+2\left(1-2 h^{2}\right) k^{\prime 2} .
$$

The scaling argument made earlier in the context of the left sphere $\mathcal{X}_{(h ; k, \beta)}$ may also be used here exactly similarly to investigate the $\rho^{\prime} \rightarrow \infty$ limit for a finite value of $k^{\prime}$. The 
representation of the relevant space $\mathcal{Y}_{(h ; 0,1)}$ in this limit reads:

$$
\begin{aligned}
\mathrm{y}_{-1} & =a c+\frac{1}{2} h c^{2}, \\
\mathrm{y}_{0} & =1+2 b c+h a c+h c d-\frac{1}{2} h^{2} c^{2}, \\
\mathrm{y}_{1} & =2 b d+h d^{2}-h-\frac{3}{2} h^{2} a c-\frac{3}{4} h^{3} c^{2} .
\end{aligned}
$$

For the embedding (2.18) we can also obtain from (2.7) the coproduct structure of the $\mathcal{Y}_{\left(h ; k^{\prime}, \beta^{\prime}\right)}$

$$
\triangle\left(\mathcal{Y}_{\left(h ; k^{\prime}, \beta^{\prime}\right)}\right) \subseteq \mathcal{Y}_{\left(h ; k^{\prime}, \beta^{\prime}\right)} \otimes \operatorname{Fun}_{h}(S L(2)) .
$$

The quantum spaces $\mathcal{X}_{(h ; k, \beta)}$ and $\mathcal{Y}_{\left(h ; k^{\prime}, \beta^{\prime}\right)}$ are isomorphic to each other in the sense that an invertible map exists so that the defining properties (2.10) and (2.13) of the algebras $\mathcal{X}_{(h ; k, \beta)}$ and $\mathcal{Y}_{\left(h ; k^{\prime}, \beta^{\prime}\right)}$ respectively are transformed to each other. The map $\pi$ reads

$$
\begin{gathered}
\pi\left(\mathrm{x}_{-1}\right)=\mathrm{y}_{1}+2 h \mathrm{y}_{0}+4 h^{2} \mathrm{y}_{-1}, \quad \pi\left(\mathrm{x}_{0}\right)=\mathrm{y}_{0}+2 h \mathrm{y}_{-1}, \quad \pi\left(\mathrm{x}_{1}\right)=\mathrm{y}_{-1}, \\
\pi(k)=-k^{\prime}, \quad \pi(\beta)=\beta^{\prime} .
\end{gathered}
$$

The inverse of the map (2.22) may be readily expressed.

\section{An infinitesimal characterization of the Jordanian quantum sphere}

In this section we give a description of the embedding of the algebras $\mathcal{X}_{(h ; k, \beta)}$ and $\mathcal{Y}_{\left(h ; k^{\prime}, \beta^{\prime}\right)}$ in the function algebra $\operatorname{Fun}_{h}(S L(2))$, as obtained in (2.14) and (2.18) respectively, by using the infinitesimal invariance of the genarating elements with respect to the appropriate twisted primitive elements of the corresponding dual universal enveloping algebra $U_{h}(s l(2))$. This approach was first developed by Koornwinder [6] in the context of the Podles quantum sphere [1] for the standard $q$-deformed function algebra $F u n_{q}(S L(2))$. For our purpose, we first recall certain definitions [6] regarding dual Hopf algebras.

Two Hopf algebras $\mathcal{U}$ and $\mathcal{A}$ are said to be in duality if there is a doubly nondegenerate bilinear form $(\mathrm{u}, \mathrm{a}) \mapsto\langle\mathrm{u}, \mathrm{a}\rangle$ such that for $(\mathrm{u}, \mathrm{v}) \in \mathcal{U},(\mathrm{a}, \mathrm{b}) \in \mathcal{A}$ we have

$$
\begin{aligned}
& \langle\triangle(\mathrm{u}), \mathrm{a} \otimes \mathrm{b}\rangle=\langle\mathrm{u}, \mathrm{ab}\rangle, \quad\langle\mathrm{u} \otimes \mathrm{v}, \triangle(a)\rangle=\langle\mathrm{uv}, \mathrm{a}\rangle, \\
& \left\langle 1_{\mathcal{U}}, \mathrm{a}\right\rangle=\varepsilon_{\mathcal{A}}(\mathrm{a}), \quad\left\langle\mathrm{u}, 1_{\mathcal{A}}\right\rangle=\varepsilon_{\mathcal{U}}(\mathrm{u}), \quad\langle S(\mathrm{u}), \mathrm{a}\rangle=\langle\mathrm{u}, S(\mathrm{a})\rangle .
\end{aligned}
$$

Instead of $\langle\mathbf{u}, \mathrm{a}\rangle$ we will also write $\mathbf{u}(\mathrm{a})$ or $\mathbf{a}(\mathbf{u})$. An element $\mathbf{u}$ of the Hopf algebra $\mathcal{U}$ is called twisted primitive with respect to a group-like element $\mathrm{g}$ of $\mathcal{U}$ if

$$
\triangle(\mathrm{u})=\mathrm{u} \otimes \mathrm{g}+\mathrm{g}^{-1} \otimes \mathrm{u}, \quad \varepsilon(\mathrm{u})=0, \quad S(\mathrm{u})=-\mathrm{gug}^{-1} .
$$


These twisted primitive elements play a key role in our present argument. For the elements $\mathrm{u} \in \mathcal{U}$ and $\mathrm{a} \in \mathcal{A}$, where $\mathcal{U}$ and $\mathcal{A}$ are Hopf algebras in duality, we define elements a.u and u.a of $\mathcal{A}$ by

$$
\mathrm{u} \cdot \mathrm{a}=(\mathrm{id} \otimes \mathrm{u})(\triangle(\mathrm{a})), \quad \mathrm{a} \cdot \mathrm{u}=(\mathrm{u} \otimes \mathrm{id})(\triangle(\mathrm{a})) .
$$

The operations defined in (3.3) are left, respectively, right algebra actions of $\mathcal{U}$ of $\mathcal{A}$. Assuming the standard notation

$$
\triangle(\mathrm{u})=\sum_{(\mathrm{u})} \mathrm{u}_{(1)} \otimes \mathrm{u}_{(2)}
$$

we now obtain

$$
\mathrm{u} \cdot(\mathrm{ab})=\sum_{(\mathrm{u})}\left(\mathrm{u}_{(1)} \cdot \mathrm{a}\right)\left(\mathrm{u}_{(2)} \cdot \mathrm{b}\right), \quad(\mathrm{ab}) \cdot \mathrm{u}=\sum_{(\mathrm{u})}\left(\mathrm{a} \cdot \mathrm{u}_{(1)}\right)\left(\mathrm{b} \cdot \mathrm{u}_{(2)}\right)
$$

for the elements $\mathrm{u} \in \mathcal{U}$ and $(\mathrm{a}, \mathrm{b}) \in \mathcal{A}$. We call an element $\mathrm{a} \in \mathcal{A}$ left (right) invariant with respect to an element $\mathrm{u} \in \mathcal{U}$ if we have $\mathbf{u} . \mathrm{a}=\varepsilon(\mathrm{u}) \mathrm{a}$, respectively, a.u $=\varepsilon(\mathrm{u}) \mathrm{a}$. The unit element of $\mathcal{A}$ is bi-invariant with respect to all $\mathrm{u} \in \mathcal{U}$. If $\mathrm{u}$ is a twisted primitive element, then $\varepsilon(\mathbf{u})=0$, and it follows from (3.5)

$$
\begin{array}{cccc}
\text { u.a }=0 \quad \text { and } \quad \text { u.b }=0 \quad & \Longrightarrow & \text { u. }(a b)=0, \\
\text { a.u }=0 \quad \text { and } \quad \text { b.u }=0 & \Longrightarrow & (a b) \cdot u=0 .
\end{array}
$$

As evidenced from (3.6) the left (right) invariant elements of $\mathcal{A}$ with respect to some twisted primitive element of $\mathcal{U}$ form a unital subalgebra of $\mathcal{A}$.

In our context, the Jordanian Hopf algebras $U_{h}(s l(2))$ and $F u n_{h}(S L(2))$ are in duality with the following pairing of the generators:

$$
\begin{aligned}
& \left\langle T^{ \pm 1},\left(\begin{array}{ll}
a & b \\
c & d
\end{array}\right)\right\rangle=\left(\begin{array}{cc}
1 & \pm h \\
0 & 1
\end{array}\right), \quad\left\langle Y,\left(\begin{array}{ll}
a & b \\
c & d
\end{array}\right)\right\rangle=\left(\begin{array}{ll}
0 & 0 \\
1 & 0
\end{array}\right), \\
& \left\langle H,\left(\begin{array}{ll}
a & b \\
c & d
\end{array}\right)\right\rangle=\left(\begin{array}{cc}
1 & 0 \\
0 & -1
\end{array}\right) .
\end{aligned}
$$

From the coalgebraic structure (2.2) of the Hopf algebra $U_{h}(s l(2))$ we see that any linear combination of $\left(T-T^{-1}\right), Y$ and $H$ is twisted primitive with respect to the group-like element $T$ of the universal enveloping algebra $U_{h}(s l(2))$. The embedding of the algebras $\mathcal{X}_{(h ; k, \beta)}$ and $\mathcal{Y}_{\left(h ; k^{\prime}, \beta^{\prime}\right)}$ in the function algebra $\operatorname{Fun}_{h}(S L(2))$ may now be naturally obtained using the previously described invariance properties with respect to these twisted primitive elements. The generators $\left(\mathbf{x}_{\mathbf{i}} \mid \mathbf{i}=-1,0,1\right)$ of the algebra $\mathcal{X}_{(h ; k, \beta)}$ are left invariant with respect to a twisted primitive element of the dual algebra $U_{h}(s l(2))$ :

$$
\mathcal{P}_{\mathcal{L}} \cdot \mathrm{x}_{\mathrm{j}}=0 \quad \text { for } \mathrm{j}=(-1,0,1)
$$


where

$$
\mathcal{P}_{\mathcal{L}}=k \rho^{-1}\left(1+\frac{3}{2} h^{2}\right)\left(\frac{T-T^{-1}}{2 h}\right)-H+2 k \rho^{-1} Y .
$$

Similarly the generators $\left(y_{i} \mid i=-1,0,1\right)$ of the algebra $\mathcal{Y}_{\left(h ; k^{\prime}, \beta^{\prime}\right)}$ are right invariant with respsect to another twisted primitive element $\mathcal{P}_{\mathcal{R}}$ of the $U_{h}(s l(2))$ algebra. This leads to an embedding of the $\mathcal{Y}_{\left(h ; k^{\prime}, \beta^{\prime}\right)}$ algebra $\operatorname{Fun}_{h}(S L(2))$. The above condition of right invarinnce reads

$$
\mathrm{y}_{\mathrm{j}} \cdot \mathcal{P}_{\mathcal{R}}=0 \quad \text { for } \quad \mathrm{j}=(-1,0,1),
$$

where the corresponding twisted primitive element is

$$
\mathcal{P}_{\mathcal{R}}=k^{\prime} \rho^{\prime-1}\left(1-\frac{1}{2} h^{2}\right)\left(\frac{T-T^{-1}}{2 h}\right)-H+2 k^{\prime} \rho^{\prime-1} Y .
$$

It is interesting to point out that the twisted primitive elements $\mathcal{P}_{\mathcal{L}}$ and $\mathcal{P}_{\mathcal{R}}$ implementing the left and the right invariances of the algebras $\mathcal{X}_{(h ; k, \beta)}$ and $\mathcal{Y}_{\left(h ; k^{\prime}, \beta^{\prime}\right)}$ respectively, are, in general,distinct. Except of the special values of the parameters $k \rho^{-1} \rightarrow 0, k^{\prime} \rho^{\prime-1} \rightarrow 0$, the elements $\mathcal{P}_{\mathcal{L}}$ and $\mathcal{P}_{\mathcal{R}}$ defined by (3.9) and (3.11) respectively, differ for a nonzero value of the deformation parameter $h$. These twisted primitives, however, become identical in the $\rho \rightarrow \infty$ and $\rho^{\prime} \rightarrow \infty$ limits discussed earlier in the context of equations (2.16) and (2.20) respectively. In these limits we obtain

$$
\left(\mathcal{P}_{\mathcal{L}}\right)_{\rho \rightarrow \infty} \sim H, \quad\left(\mathcal{P}_{\mathcal{R}}\right)_{\rho^{\prime} \rightarrow \infty} \sim H
$$

\section{Conclusion}

In conclusion, we have constructed a one parameter family of algebras invariant under the left (right) coactions of the group-like elements $\mathcal{T}_{h}^{(j=1)}$ of the Jordanian function algebra $\operatorname{Fun}_{h}(S L(2))$. These algebras may be regarded as Jordanian quantization of the twodimensional spheres. Algebraically, an invertible map exist between the quantum spaces invariant under the left and the right coactions of the $\mathcal{T}_{h}^{(j=1)}$ matrix. The embedding of these algebras in the function algebra $\operatorname{Fun}_{h}(S L(2))$ may be understood as an infinitesimal invariance of the generating elements of these algebras with respect to the appropriate twisted primitive elements of the Jordanian enveloping algebra $U_{h}(\operatorname{sl}(2))$. It is interesting to point out that, unlike the standard $q$-deformed case [6], in the present Jordanian example the twisted primitive elements $\mathcal{P}_{\mathcal{L}}$ and $\mathcal{P}_{\mathcal{R}}$ associated with the spaces invariant under the left and the coactions of the $\mathcal{T}_{h}^{(j=1)}$ matrix respectively, are distinct except for special values of the parameters.

As applications and further extensions of the present work, we wish consider the followings. The classification of the differential calculi on the Jordanian deformed sphere may be obtained. We will present the results elsewhere. In another development, the authors of the 
Ref. [18] considered the invariant propagator of the $h$-deformed Laplacian on the $h$-deformed Lobachevski plane. Now this propagator may be studied on the Jordanian spherical spaces. Lastly, we have recently constructed [23] a Jordanian deformed quasi-Hopf enveloping algebra satisfying a shifted Yang-Baxter equation. The present problem may be investigated in the framework of Jordanian quasi-Hopf function algebra.

\section{Acknowledgement}

We wish to thank Amitabha Chakrabarti for fruitful correspondence.

\section{References}

[1] P. Podles, Lett. Math. Phys. 14, 193 (1987)

[2] P. Podles, Lett. Math. Phys. 18, 107 (1989)

[3] P. Podles, Comm. Math. Phys. 150, 167 (1992)

[4] J. Apel and J. Schmudgen, Lett. Math. Phys. 32, 25 (1994)

[5] M. Noumi and K. Mimachi, Comm. Math. Phys. 128, 521 (1990)

[6] T. H. Koornwinder, SIAM J. Math. Anal. 24, 795 (1993)

[7] B. A. Kupershmidt, J. Phys. A25, L1239 (1992)

[8] E.E. Demidov, Yu. I. Manin, E. E. Mukhin, D. V. Zhadanovich, Prog. Theo. Phys. Suppl. 102, 203 (1990)

[9] Ch. Ohn, Lett. Math. Phys. 25, 85 (1992).

[10] A. Aghamohammadi, Mod. Phys. Lett. A8, 2607 (1993)

[11] A. D. Jacobs and J. F. Cornwall J. Phys. A31, 8869 (1998)

[12] A. Aghamohammadi, M. Khorrami and A. Shariati, J. Phys. A28, L225 (1995)

[13] B. Abdesselam, A. Chakrabarti and R. Chakrabarti, Mod. Phys. Lett. A13, 779 (1998)

[14] R. Chakrabarti and C. Quesne, Int. J. Mod. Phys. A14, 2511 (1999).

[15] T. Filk, Phys. Lett. B376, 53 (1996)

[16] H. Grosse, C. Klymick, P. Presnajder, Int. J. Theo. Phys. 35, 231 (1996)

[17] S. Minwalla, M. V. Ramsdonk, N. Seiberg, Noncommutative perturbative dynamics, hep-th /9912072 
[18] J. Madore and H. Steinacker, J. Phys. A33, 327 (2000)

[19] H. Grosse, J. Madore and H. Steinacker, Field theory on the q-deformed fuzzy sphere I, hep-th/0005273

[20] M.Douglas and C. Hull, JHEP 9802, 008 (1998)

[21] C. S. Chu and P. M. Ho, Nucl. Phys. B550, 151 (1999)

[22] N. Seiberg and E. Witten, JHEP 9909, 032 (1999)

[23] A. Chakrabarti and R. Chakrabarti, J. Phys. A33, 4611 (2000) 